\title{
Correction to: Exploring the molecular insights of concurrent composite mucoepidermoid carcinoma and papillary thyroid carcinoma
}

\author{
Rosa Falcone ${ }^{1}$ - Marialuisa Sponziello $\mathbb{1}^{1}$ - Raffella Carletti ${ }^{2}$ Cira Di Gioia ${ }^{2}$ - Francesco Nardi $^{2} \cdot$ Catia Mio $^{3}$. \\ Valeria Pecce ${ }^{1}$. Luana Abballe ${ }^{1} \cdot$ Giorgio Grani $^{1} \cdot$ Valeria Ramundo $^{1} \cdot$ Giuseppe Damante $^{3} \cdot$ Cosimo Durante $^{1}$. \\ Marco Filetti ${ }^{4} \cdot$ Michela Roberto $^{4} \cdot$ Paolo Marchetti $^{2} \cdot$ Antonella Verrienti $^{1}$
}

Published online: 23 March 2020

(c) Springer Science+Business Media, LLC, part of Springer Nature 2020

\section{Correction to: Endocrine}

https://doi.org/10.1007/s12020-020-02221-8

The original version of this article unfortunately contained a mistake in the author names. The given name and the family name have been interchanged for all the authors in the article.
The corrected authors' group has been given below:

Rosa Falcone, Marialuisa Sponziello, Raffella Carletti, Cira Di Gioia, Francesco Nardi, Catia Mio, Valeria Pecce, Luana Abballe, Giorgio Grani, Valeria Ramundo, Giuseppe Damante, Cosimo Durante, Marco Filetti, Michela Roberto, Paolo Marchetti and Antonella Verrienti.

The original article has been corrected.

The original article can be found online at https://doi.org/10.1007/ s12020-020-02221-8.

Marialuisa Sponziello

marialuisa.sponziello@uniroma1.it

1 Department of Translational and Precision Medicine, "Sapienza" University of Rome, 00185 Rome, Italy

2 Department of Radiological, Oncological and Pathological Sciences, "Sapienza" University of Rome, Rome, Italy

3 Department of Medicine, University of Udine, 33100 Udine, Italy

4 Department of Clinical and Molecular Medicine, Sant'Andrea Hospital, "Sapienza" University of Rome, 00189 Rome, Italy 